\title{
Colorimetric Strategy for Highly Sensitive and Selective Simultaneous Detection of Histidine and Cysteine Based on G-Quadruplex-Cu(II) Metalloenzyme
}

Changtong Wu, ${ }^{\dagger}, \S$ Daoqing Fan, ${ }^{\dagger}$ Chunyang Zhou, ${ }^{\dagger}$ Yaqing Liu, ${ }^{*}$, Erkang Wang ${ }^{*}, \dagger$

${ }^{\dagger}$ State Key Laboratory of Electroanalytical Chemistry, Changchun Institute of Applied Chemistry, Chinese Academy of Sciences, Changchun, Jilin 130022, China

${ }^{\star}$ Key Laboratory of Food Nutrition and Safety, Tianjin University of Science and Technology, Ministry of Education, Tianjin 300457, China

${ }^{\S}$ Department of Chemistry and Environmental Engineering, Changchun University of Science and Technology, Changchun, Jilin 130022, China

\author{
*Corresponding Author \\ E-mail: yaqingliu@ tust.edu.cn (Y. Liu); ekwang@ciac.ac.cn (E. Wang) \\ Tel: +86-431-85262003. Fax: +86-431-85689711.
}




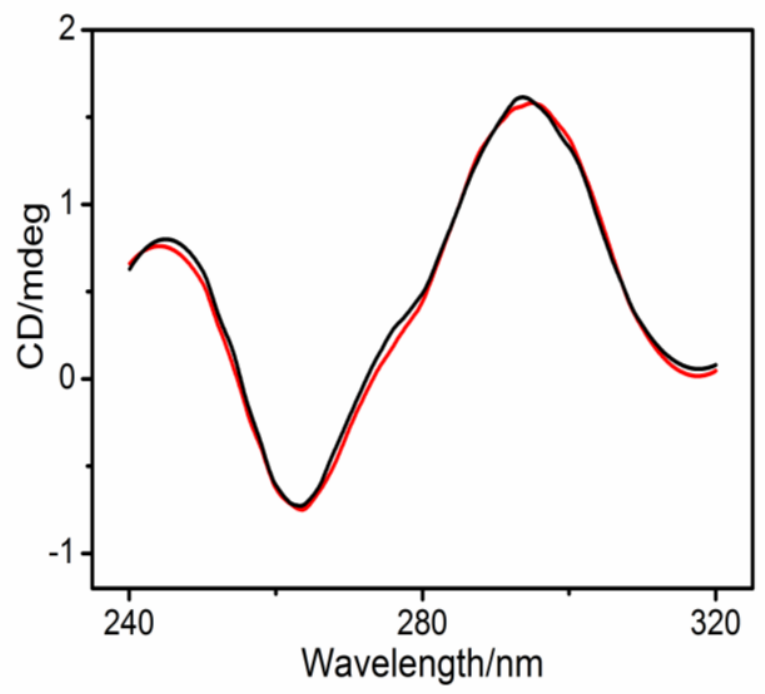

Figure S1 The Circular dichroism (CD) spectra of G-quadruplex (black curve) and G-quadruplex-Cu complex (red curve) in HEPES buffer (20 mM HEPES, $150 \mathrm{mM}$ $\mathrm{NaCl}, \mathrm{pH} 7.0)$. [G-quadruplex $]=5 \mathrm{uM},\left[\mathrm{Cu}^{2+}\right]=75 \mathrm{uM}$.
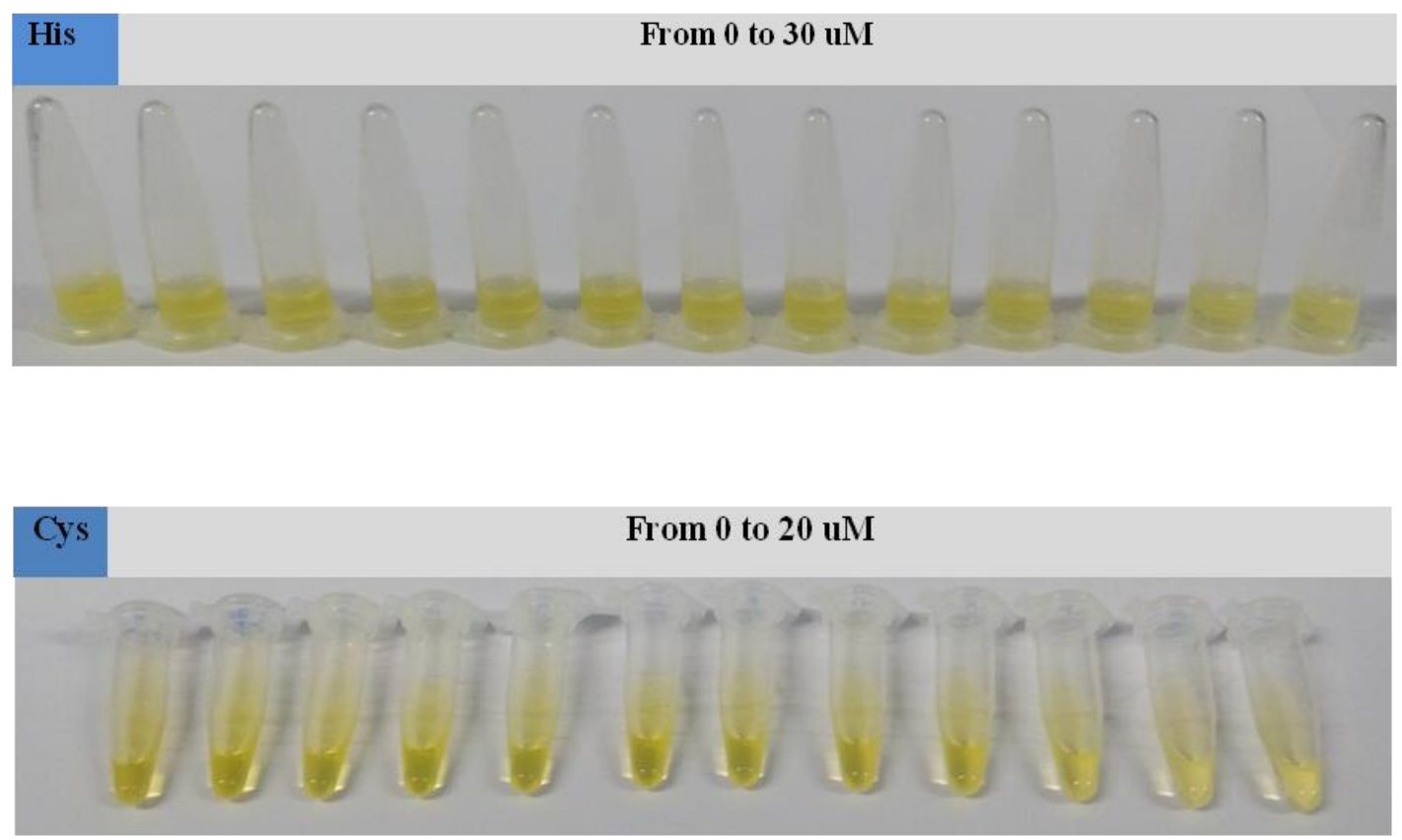

Figure S2 The photos of coloric changes with different concentrations of histidine (top) and cysteine (bottom). 
Table S1. Comparision of the LOD for histidine with other colorimetric arrays

\begin{tabular}{|c|c|c|}
\hline Arrays & LOD & Reference \\
\hline Julolidine moisty based compound- $\mathrm{Cu}$ array & $9.0 \mathrm{uM}$ & 1 \\
\hline Quercetin-functionalized gold nanoparticles & $30 \mathrm{nM}$ & 2 \\
\hline An indicator-displacement array & $400 \mathrm{nM}$ & 3 \\
\hline $\mathrm{Cu}^{2+}$-ligand complex array & $2 \mathrm{uM}$ & 4 \\
\hline Lysine-functionalized silver nanoparticles & $5 \mathrm{uM}$ & 5 \\
\hline Cysteine modified Ag nanoparticles & $0.3 \mathrm{uM}$ & 6 \\
\hline AuNP-calix assembly & $2 \mathrm{uM}$ & 7 \\
\hline DNAzyme cascade & $500 \mathrm{uM}$ & 8 \\
\hline Our work & $10 \mathrm{nM}$ & \\
\hline
\end{tabular}


Table S2. Comparision of the LOD for cysteine with other colorimetric arrays

\begin{tabular}{|c|c|c|}
\hline Arrays & LOD & Reference \\
\hline Julolidine moisty based compound-Cu array & $3.6 \mathrm{uM}$ & 1 \\
\hline G-quadruplex DNAzyme-based cysteine sensor & $19 \mathrm{nM}$ & 9 \\
\hline Polythiophene derivative-based colomeric and & $50 \mathrm{nM}$ & 10 \\
\hline fluorescent dual probe & $64 \mathrm{nM}$ & 11 \\
\hline G-quadruplex-hemin DNAzymes disruption by ${ }^{+}$system & $40 \mathrm{nM}$ & 12 \\
\hline Silver-ion-mediated DNAzyme system & $0.1 \mathrm{uM}$ & 13 \\
\hline ssDNA-stabilized gold nanoparticles as probe & $100 \mathrm{nM}$ & 14 \\
\hline DNA-AuNPs based colorimetic competition array & $5 \mathrm{nM}$ & \\
\hline Our work & & \\
\hline
\end{tabular}




\section{References}

(1) Kim, Y. S.; Park, G. J.; Lee, S. A.; Kim, C. RSC Adv. 2015, 5, 31179-31188.

(2) Rawat, K. A.; Kailasa, S. K. Microchim. Acta 2014, 181, 1917-1929.

(3) Sun, S. K.; Tu, K. X.; Yan, X. P. Analyst 2012, 137, 2124-2128.

(4) Zhang, S.; Yang, C.; Zhu, W.; Zeng, B.; Yang, Y.; Xu, Y.; Qian, X. Org. Biomol. Chem. 2012, $10,1653-1658$.

(5) Bae, D. R.; Han, W. S.; Lim, J. M.; Kang, S.; Lee, J. Y.; Kang, D.; Jung, J. H. Langmuir 2010, 26, 2181-2185.

(6) Li, H.; Bian, Y. Nanotechnology 2009, 20, 1-6.

(7) Patel, G.; Menon, S. Chem. Commun. 2009, 3563-3565.

(8) Elbaz, J.; Shlyahovsky, B.; Willner, I. Chem. Commun. 2008, 1569-1571.

(9) Jia, S. M.; Liu, X. F.; Li, P.; Kong, D. M.; Shen, H. X. Biosens. Bioelectron. 2011, 27, $148-152$.

(10) Yao, Z.; Bai, H.; Li, C.; Shi, G. Chem. Commun. 2011, 47, 7431-7433.

(11) Zhou, X. H.; Kong, D. M.; Shen, H. X. Anal. Chem. 2010, 82, 789-793.

(12) Li, T.; Shi, L.; Wang, E.; Dong, S. Chemistry 2009, 15, 3347-3350.

(13) Chen, Z.; Luo, S.; Liu, C.; Cai, Q. Anal. Bioanal. Chem. 2009, 395, 489-494.

(14) Jae-Seung, L.; Pirmin A., U.; Min Su, H.; Chad A., M. Nano Lett. 2008, 8, 529-533. 